\title{
Clostridioides difficile Infection and Liver Cirrhosis - A Retrospective, Cohort Study
}

\author{
Nikhitha Mantri' \\ Harish Patel (D) 1,2 \\ Kanthi Rekha Badipatla ${ }^{1,2}$ \\ Haozhe Sun' \\ Danial Shaikh (D) ${ }^{1,2}$ \\ Sudharsan Gongati ${ }^{\prime}$ \\ Suresh Kumar Nayudu ${ }^{1,2}$ \\ 'Department of Medicine, Bronx Care \\ Health System, Affiliated with Icahn \\ School of Medicine at Mount Sinai, Bronx, \\ NY, USA; '2Division of Gastroenterology, \\ Bronx Care Health System, Affiliated \\ with Icahn School of Medicine at Mount \\ Sinai, Bronx, NY, USA
}

Purpose: Clostridioides difficile infection (CDI) is one of the most common health careassociated infections in the United States. Studies revealed a higher mortality when CDI is associated with liver cirrhosis. We aim to present the outcomes of CDI among patients with and without liver cirrhosis and to analyze the association of Model for End-Stage Liver Disease (MELD) and Child-Pugh (CPT) scoring with the severity of CDI.

Methods: A retrospective observational study was conducted in hospitalized patients with CDI diagnosed via a 2-step method - glutamate dehydrogenase (GDH) and toxin polymerase chain reaction (PCR). Patients with liver cirrhosis were identified based on ICD codes and chart review. MELD and CPT scores were calculated using laboratory parameters at the time of hospitalization. We compared CDI-related mortality in patients with and without cirrhosis and reviewed the CDI severity distribution in cirrhosis patients.

Results: A total of 526 patients were included in the study, of which 478 (90.87\%) were non-cirrhotic and $48(9.13 \%)$ were cirrhotic patients. Mortality rate was higher in cirrhosis group compared to the non-cirrhosis group $(39.6 \%$ vs. $14.6 \%, \mathrm{P}=0.001)$. Among cirrhosis patients, those who survived had lower MELD score compared to the expired group (14.9 vs. $18.58, \mathrm{P}=0.106)$. There was no correlation of mortality based on CPT score in the cirrhosis group $(\mathrm{P}=0.062)$. In post hoc analysis comparing the severity of CDI to liver cirrhosis, cirrhosis patients are more likely to present with severe-complicated disease. Multivariate logistic regression identified liver cirrhosis, severe-complicated CDI and serum albumin level as independent predictors of mortality.

Conclusion: Our study noted a more severe disease presentation and higher mortality in patients with cirrhosis admitted with CDI. Further studies are required for better understanding of the clinical course of CDI in cirrhosis and to evaluate the need for early intervention in this patient group.

Keywords: clostridioides difficile infection; CDI, cirrhosis, mortality, severity, Model for End-Stage Liver Disease; MELD, Child-Pugh; CPT

\section{Introduction}

Clostridioides difficile infection (CDI) is an anaerobic, spore-producing gram positive bacterium. The infection can range anywhere from being an asymptomatic carrier to fulminant colitis. ${ }^{1}$ CDI is one of the most common causes of health care-associated diarrhea in the United States. ${ }^{2}$ There are around 453,000 patients diagnosed with a new CDI with an estimated cure 30 day mortality of $9.3 \%{ }^{3}$ CDI has a significant health care expenditure with estimated annual health care costs of $\$ 4.8$ billion $^{4}$ and there was has been significant increment in managing the primary and recurrent CDI. ${ }^{5}$

Severity scores consist of the attributes of inflammatory markers of CDI-related sepsis as depicted by the albumin, leukocytosis, renal injury or requirement of the
Correspondence: Suresh Kumar Nayudu Bronx Care Health System, Affiliated with Icahn School of Medicine at Mount Sinai, 1650 Selwyn Ave, Bronx, NY, USA

Tel + I 2122035476

Fax + I 7189602055

Email SNAYUDU@bronxleb.org 
critical care monitoring. The CDI severity is classified based on crude 30-day mortality or need for the colectomy. ${ }^{6}$ However, other parameters like liver cirrhosis, which could contribute to higher mortality, have not been accounted in decision algorithm for CDI management.

Use of antibiotics and proton pump inhibitors are some of the well-known risk factors for CDI. ${ }^{7}$ In lieu of the antibiotic use for sub-acute bacterial peritonitis prophylaxis and proton pump inhibitor for gastrointestinal bleeding, the prevalence of the CDI is higher in patient with liver cirrhosis. ${ }^{8}$ Based on the Nationwide Inpatient Sample (NIS) database, the CDI-related mortality is higher in patient with liver cirrhosis compared those no cirrhosis (13.8\% vs. $8.2 \%){ }^{9}$ Most of the studies regarding CDI in cirrhotic patient is from the National patient database. ${ }^{9,10}$ Hence, there is limited data on the severity of CDI in patient with liver cirrhosis and impact of liver cirrhosis severity on outcome of the CDI.

In our retrospective study we evaluated the impact of liver cirrhosis on CDI outcome of mortality including comparison of variance in the CDI severity in patient with liver cirrhosis and no cirrhosis. We also wanted to see if the liver cirrhosis severity factors, like Model for End-Stage Liver Disease (MELD) score or the Child-Pugh (CPT) Score, had any impact on the CDI severity.

\section{Materials and Methods}

Our study is a retrospective review of all hospitalized patients at BronxCare Health System. The study protocol has been reviewed and approved by institutional Internal Review Board (IRB) of Bronx Care Health System - IRB number \# 051117 05. Patient's consent was not required by the IRB as the study is retrospective. The patient data accessed complied with relevant data protection and privacy regulations. The study was performed as per the Declaration of Helsinki.

\section{Study Population}

The study population includes all hospitalized patients above the age of 18 admitted with or diagnosed with CDI during the hospital stay. We include patients admitted from July 1, 2014 to December 30, 2017. Diagnosis of CDI was established with a 2-step method: positive GDH and positive stool Cepheid GeneXpert ${ }^{\circledR}$ PCR test for toxin $\mathrm{A}$ and $\mathrm{B}$ in patients with clinical features suggestive of $\mathrm{CDI}$ at any point during the hospital stay.

\section{Liver Cirrhosis}

The diagnosis of liver cirrhosis was established through abstraction of ICD-10 and ICD-9 coding in the electronic medical record (EMR). Diagnosis of liver cirrhosis was confirmed through the chart review. The MELD and CPT scores were based on the laboratory parameters at time of hospitalization.

\section{Severity}

We drafted a protocol to classify the CDI severity based on the recommendation drafted by American College of Gastroenterology, which was in affect at the time of the management of patients. The severity for the CDI were graded into three categories: mild to moderate, severe, and severe to complicated category. ${ }^{11}$

\section{Laboratory Parameters and Co-Morbid Medical Conditions}

We abstracted the demographic details, comorbidities, laboratory tests, radiographic test results, review of the medical charts and medication history. The comorbidities abstracted using the ICD-10 and ICD-9 codes from the EMR. Laboratory parameters used to gauge the severity of CDI were abstracted from the day of the collection of the stool specimen for the CDI stool toxin.

\section{Study Outcome}

The primary outcome of the study was to compare the mortality during the hospitalization in patient with CDI and liver cirrhosis vs. those with no liver cirrhosis. We also wanted to review the CDI severity distribution for the patients with liver cirrhosis vs. with no liver cirrhosis. We intended to further evaluate if the liver cirrhosis severity impacted the mortality in patients with CDI.

\section{Statistical Analysis}

We performed the statistical analysis with IBM SPSS (Statistical Packages for the Social Sciences) version 19. For comparing the baseline characteristics, we used frequencies and percentages for categorical variable, for the numerical continuous variables we used mean and standard deviation. We used chi-square analysis with Pearson test for the dichotomous variables and the Student's $t$-test for the for the continuous variable. A twotailed value of $<0.05$ was considered statistically significant. We performed the regression analysis to compute the statistically significant independent predictors of the 
mortality. One-way ANOVA and post-hoc analysis were used to evaluate the severity distribution of CDI in patients with liver cirrhosis vs. those with no liver cirrhosis and adjusted residual of above 1.8 was considered significant. The normality test was performed and variable with the skewed deviation were presented as median and interquartile range. The comparison for the same is performed with Mann-Whitney $U$-test. We performed the regression analysis for analyzing the predictor of mortality in patients with CDI. A log rank test is used to analyze the mortality distribution amongst the patient with liver cirrhosis and no liver cirrhosis and results are represented with Kaplan-Meier curves.

\section{Results}

A total of 526 patients who were hospitalized during the study period and had a diagnosis of CDI were included in the study: 48 (9.13\%) had liver cirrhosis and 478 (90.87\%) had no cirrhosis. Table 1 describes the distribution of the patients' demographics, comorbidities and laboratory parameters of the patients included in the study.

Table I Characteristics of the Patients With and Without Liver Cirrhosis

\begin{tabular}{|c|c|c|c|}
\hline & Cirrhosis $(n=48)$ & No Cirrhosis $(n=478)$ & $p$ value \\
\hline \multicolumn{4}{|l|}{ Demog2raphics } \\
\hline \multirow[t]{2}{*}{ Age, years - mean $( \pm S D)$} & $58.94( \pm 11.01)$ & $57.60( \pm 15.79)$ & 0.567 \\
\hline & & & 0.031 \\
\hline Male Gender - n (\%) & $19(39.58)$ & $267(55.86)$ & \\
\hline Ethnicity - n (\%) & & & 0.269 \\
\hline Hispanic & $25(52.08)$ & $253(52.93)$ & \\
\hline African American & $12(25.00)$ & $148(30.96)$ & \\
\hline Caucasian & $7(14.58)$ & $33(06.90)$ & \\
\hline Others & $4(08.33)$ & $44(09.21)$ & \\
\hline \multicolumn{4}{|l|}{ Associated comorbidities - n (\%) } \\
\hline Hypertension & $38(79.17)$ & $337(70.65)$ & 0.213 \\
\hline Diabetes mellitus & $26(54.17)$ & $262(54.9)$ & 0.92 \\
\hline ESRD & $5(10.42)$ & $63(13.21)$ & 0.583 \\
\hline $\mathrm{CHF}$ & II (22.92) & $156(32.70)$ & 0.165 \\
\hline \multicolumn{4}{|l|}{ Presence of other infections $-\mathrm{n}(\%)$} \\
\hline Sepsis & $21(43.75)$ & $233(48.85)$ & 0.501 \\
\hline Pneumonia & $25(52.08)$ & 281 (58.9I) & $0.36 \mathrm{I}$ \\
\hline Urinary tract infection & $15(31.25)$ & $145(30.40)$ & 0.903 \\
\hline \multicolumn{4}{|l|}{ Laboratory parameters - mean $( \pm S D)$} \\
\hline WBC, k/ul & $|7.6|(\underline{ \pm} \mid 4.20)$ & $15.73( \pm /-9.52)$ & 0.222 \\
\hline Serum Albumin, $g / d L$ & $3.46(+0.85)$ & $2.8(+0.7)$ & 0.001 \\
\hline BUN, mg/dl & $41.88( \pm 29.51)$ & $36.09( \pm 33.14)$ & 0.246 \\
\hline Serum Creatinine, $\mathrm{mg} / \mathrm{dl}^{*}$ & $\mathrm{I} . \mathrm{I}(3)$ & $1.75(3.3)$ & 0.273 \\
\hline Serum Potassium, mEq/L & $3.26( \pm 0.63)$ & $3.36( \pm 0.7 I)$ & 0.374 \\
\hline Serum Chloride, mEq/L & $92.07( \pm 15.39)$ & $95.25( \pm 8.76)$ & 0.029 \\
\hline Serum Bicarbonate, $\mathrm{mEq} / \mathrm{L}$ & $15.04( \pm 5.44)$ & $17.43( \pm 5.53)$ & 0.005 \\
\hline Lactate, mmoles/L* & $2( \pm 2)$ & $3.17( \pm 3.63)$ & 0.22 \\
\hline \multicolumn{4}{|l|}{ Outcome } \\
\hline Mortality - n (\%) & $19(39.6 \%)$ & $2.7(3)$ & 0.15 \\
\hline Length of Stay, days - median (IQR)* & $2(6)$ & $2(5)$ & 0.0806 \\
\hline
\end{tabular}

Notes: The above data is being presented using $\mathrm{n}(\%)$ and mean \pm standard deviation; ${ }^{*}$ Continuous variable represented with Median (interquartile range) and comparison is made with the Mann-Whitney U-test.

Abbreviations: ESRD, end-stage renal disease; CHF, congestive heart failure; WBC, white blood cell; BUN, blood urea nitrogen. 
The non-cirrhosis group had a higher percentage of males than females $(55.86 \%$ vs. $44.14 \%$; $\mathrm{P}=0.031)$. We assessed various comorbidities like hypertension, diabetes, end-stage renal disease (ESRD) and congestive heart failure (CHF); no statistical difference was observed between the groups.

Among the laboratory parameters, the differences in the mean albumin, mean chloride and mean bicarbonate levels were statistically significant. The mean albumin levels were higher in the cirrhosis group compared to the non-cirrhosis group $(3.46 \mathrm{mg} / \mathrm{dl}$ vs. $2.8 \mathrm{mg} / \mathrm{dl} ; \mathrm{P}=0.001)$ The mean chloride levels among the cirrhosis group and the non-cirrhosis group were $95.07 \mathrm{mEq} / \mathrm{L}$ vs. $92.25 \mathrm{mEq} /$ $\mathrm{L}(\mathrm{p}=0.029)$. The mean bicarbonate levels were 15.04 $\mathrm{mEq} / \mathrm{L}$ vs. $17.43 \mathrm{mEq} / \mathrm{L}(\mathrm{p}=0.005)$ respectively.

The overall mortality rate for the patients hospitalized with the CDI was $16.9 \%$ (89/526), of which cirrhotic patients had a higher mortality rate compared to the noncirrhosis patients, $39.6 \%$ vs. $14.6 \%$, respectively $(\mathrm{P}=$ 0.001). The patients with liver cirrhosis had shorter length of stay of 4.19, as compared to 6.08 days in patients with no liver cirrhosis, however this difference was not statistically significant. A log rank test determined that patients with liver cirrhosis had a statistically different survival pattern than those with no liver cirrhosis $(\mathrm{P}<0.001)$. This comparison in survival can be seen in the KaplanMeier curves in Figure 1.

A one-way ANOVA and post-hoc analysis (Table-2), revealed that patients with liver cirrhosis are more likely to have severe presentation of CDI $(43.8 \%, \mathrm{n}=21)$, whereas mild CDI $(70.7 \%, \mathrm{n}=338)$ was the most common severity presentation in patients with no liver cirrhosis. This distribution was statistically significant, with $\mathrm{P}=0.001$.

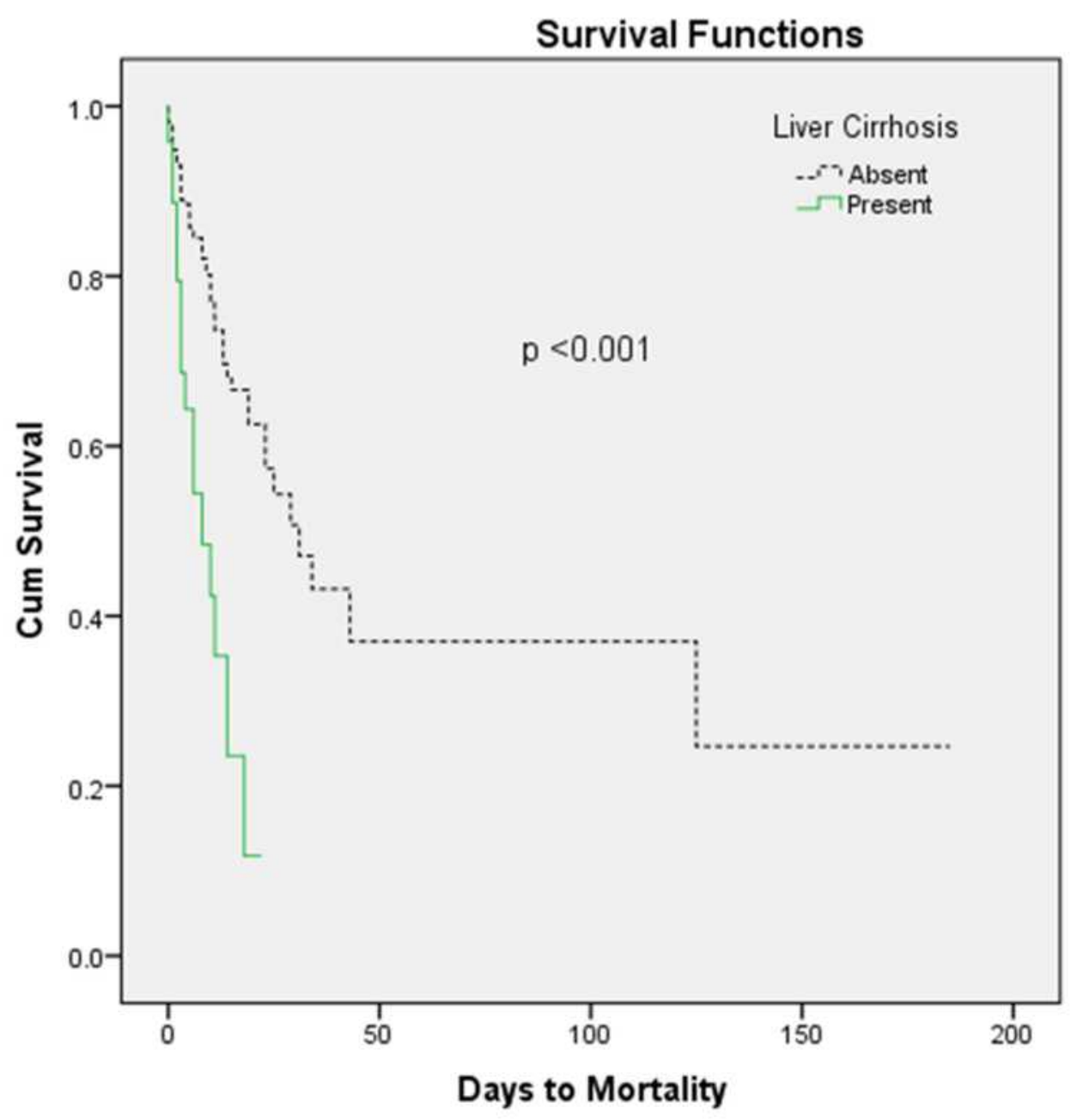

Figure I Kaplan-Meier curves. 
Table 2 Post Hoc Analysis of CDI Severity and Liver Cirrhosis

\begin{tabular}{|c|c|c|c|c|}
\hline & $\begin{array}{l}\text { Mild- } \\
\text { Moderate } \\
\text { CDI }\end{array}$ & $\begin{array}{l}\text { Severe } \\
\text { CDI }\end{array}$ & $\begin{array}{l}\text { Severe- } \\
\text { Complicated } \\
\text { CDI }\end{array}$ & $\begin{array}{l}P= \\
0.001\end{array}$ \\
\hline $\begin{array}{l}\text { Liver Cirrhosis } \\
\text { Present - n (\%) }\end{array}$ & $18(37.5)$ & $9(18.8)$ & $21(43.8)$ & \\
\hline Adjusted Residual & -4.7 & 0.1 & 6.3 & \\
\hline $\begin{array}{l}\text { Liver Cirrhosis } \\
\text { Absent - n (\%) }\end{array}$ & $338(70.7)$ & $88(18.4)$ & $52(10.9)$ & \\
\hline Adjusted Residual & 4.7 & -.1 & -6.3 & \\
\hline
\end{tabular}

Table 3 Binomial Logistic Regression of Predictors of Mortality

\begin{tabular}{|l|l|l|}
\hline Variable & Odds Ratio & p-value \\
\hline Age & I.0I (0.985-I.02) & 0.49 \\
Gender (Male) & $1.08(0.63-1.87)$ & $0.8 \mathrm{I}$ \\
Liver Cirrhosis & $0.55(0.255-0.86))$ & 0.047 \\
Severe CDI & $1.7(0.845-3.53)$ & 0.134 \\
Severe to Complicated CDI & $6.33(3.18-13.8)$ & 0.001 \\
leukocyte count & $1.02(0.99-1.05)$ & 0.06 \\
Serum. Creatinine & $1.08(1.02-1.19)$ & 0.054 \\
Serum. Albumin & $0.485(0.343-0.686)$ & 0.001 \\
\hline
\end{tabular}

Multivariate logistic regression of predictors of mortality (Table 3 ) showed that liver cirrhosis had an odds ratio of 0.55 (95\% confidence interval $[\mathrm{CI}]=0.255-0.86 ; \mathrm{P}=$ 0.047). Patients with severe CDI and severe-complicated CDI had an odds ratio for mortality of $1.7(0.845-3.53$; $\mathrm{P}=0.134)$ and $6.33(3.18-13.8 ; \mathrm{P}=0.001)$ respectively.

The odds of elevations in leucocyte count, serum creatinine and serum albumin were $1.02 \mathrm{k} / \mathrm{ul}(0.99-1.05 ; \mathrm{P}=$ $0.06), 1.08 \mathrm{mEq} / \mathrm{L}(1.02-1.19 ; \mathrm{P}=0.054)$ and 0.485 (0.343-0.686; $\mathrm{P}=0.001)$ respectively.

Table 4 depicts the demographic and severity of liver disease distribution in patients with CDI and liver cirrhosis. Our study showed a mortality of $39.5 \%(n=19)$ in this group. Patients who expired had a mean age of 57.05 years compared to the survivors whose mean age was 60.17 years $(\mathrm{P}=0.003)$.

As for the severity of cirrhosis, both MELD and CPT scores were calculated. The MELD score of patients with liver cirrhosis and CDI who expired was higher, 18.58 ( \pm 8.73 ), for the patient who expired compared to those who survived, $14.97( \pm 6.46)$, but this difference is not statistically significant. The majority of patients who expired with the CPT-A, $52.6 \%$, as compared to $37.9 \%$ of patients with CPT-A in those who survived. The majority of the patients with CDI and the liver
Table 4 Demographic Distribution of Patients with Liver Cirrhosis and CDI

\begin{tabular}{|c|c|c|c|}
\hline & $\begin{array}{l}\text { Survived } \\
(n=29)\end{array}$ & $\begin{array}{l}\text { Expired } \\
(n=19)\end{array}$ & $p$ value \\
\hline Age, years - mean ( $( \pm S D)$ & $60.17( \pm 9.99)$ & $57.05( \pm 12.46)$ & 0.003 \\
\hline $\begin{array}{l}\text { Length of Stay, days - } \\
\text { mean ( } \pm \text { SD) }\end{array}$ & $3.66( \pm 5.03)$ & $5.00( \pm 5.06)$ & 0.371 \\
\hline Male Gender - n (\%) & $17(58.62)$ & $12(63.16)$ & 0.753 \\
\hline $\begin{array}{l}\text { Ethnicity - n (\%) } \\
\text { African American } \\
\text { Hispanic } \\
\text { Caucasian } \\
\text { Others }\end{array}$ & $\begin{array}{l}8(27.59) \\
16(55.17) \\
2(06.90) \\
3(10.34)\end{array}$ & $\begin{array}{l}4(21.05) \\
9(47.37) \\
5(26.32) \\
1(5.26)\end{array}$ & 0.301 \\
\hline $\begin{array}{l}\text { MELD score - mean } \\
( \pm S D)\end{array}$ & $14.97( \pm 6.46)$ & $18.58( \pm 8.73)$ & 0.106 \\
\hline $\begin{array}{l}\text { Child-Pugh Score - } \\
\text { mean (士SD) } \\
\text { CPT-A } \\
\text { CPT-B } \\
\text { CPT-C }\end{array}$ & $\begin{array}{l}11( \pm 37.93) \\
8( \pm 27.59) \\
10( \pm 34.48)\end{array}$ & $\begin{array}{l}10( \pm 52.63) \\
8( \pm 42.11) \\
1( \pm 5.26)\end{array}$ & 0.062 \\
\hline $\begin{array}{l}\text { C. difficile severity - } \\
\text { n (\%) } \\
\text { Mild to Moderate } \\
\text { Severe } \\
\text { Severe to Complicated }\end{array}$ & $\begin{array}{l}14(48.27) \\
5(17.24) \\
10(34.49)\end{array}$ & $\begin{array}{l}4(20.15) \\
4(20.15) \\
\text { II (57.89) }\end{array}$ & 0.15 \\
\hline
\end{tabular}

Abbreviations: MELD score, Model for End-Stage Liver Disease score; CPT, Child-Pugh Score.

cirrhosis had severe to complicated presentations of CDI $(57.8 \%, \mathrm{n}=11)$.

\section{Discussion}

Our study data confer the prior published report revealing a significantly higher mortality in patients with liver cirrhosis and CDI compared to those with no liver cirrhosis and CDI. ${ }^{9,12}$ The patients with the liver cirrhosis has high susceptibility for CDI due to altered bowel microbial flora from bacterial translocation, increased antibiotics and proton pump inhibitor use, and frequent hospitalization. ${ }^{13,14}$ However, the precise mechanism of the poor outcomes of CDI in patients with the liver cirrhosis has been poorly understood.

Patients with liver cirrhosis have a dysfunctional immune response to sepsis and tend to mount an altered inflammatory response to the inciting infection. ${ }^{15}$ Mild presentation is the most common clinically severity of CDI, and in our study $70 \%$ of patients with no liver cirrhosis did present with mild severity. However, in 
patients with liver cirrhosis clinically severe to complicated CDI remains the most common presentation, accounting for up to $43.8 \%$ of patients with liver cirrhosis.

Patients with liver cirrhosis tend to have lower levels of serum albumin and it is predictor of poor outcomes and increased mortality. ${ }^{16}$ Serum albumin levels are also used in triaging the severity of the CDI patients, hence this would explain the increment in severe to complicated CDI presentation in patients with liver cirrhosis. However, on multivariate logistic regression serum albumin level and liver cirrhosis both were independent predictors of mortality in patient with CDI. This outcomes denotes that liver cirrhosis as an independent predictor of CDI outcomes, rather than an indirect affect through hypoalbuminemia.

The more severe presentations of CDI can increase the mortality in patients with liver cirrhosis. However, multivariate logistic regression analysis did identify liver cirrhosis as an independent predictor of mortality. The CDI induced sepsis can lead to new-onset or worsening of the pre-existing decompensation of liver cirrhosis leading to high mortality rate. ${ }^{17}$ Liver cirrhosis can affect mortality in patients with CDI irrespective of the severity of the illness. Hence, it is worthwhile to evaluate for escalation of the antibiotics therapy for CDI in patients with liver cirrhosis.

Hong et al, in a study of 701 cirrhotic patients, showed that the MELD score is an independent predictor of mortality in patients with CDI and cirrhosis. In our study we noticed that patients with liver cirrhosis who survived had lower MELD scores compared to those who died from CDI (14.9 vs. 18.58), however due to the lower number of patients with liver cirrhosis and CDI, we were unable to evaluate for a statistically significant correlation. CPT scoring is also utilized to assess prognosis in patient with liver cirrhosis. The utility of prognosis scoring for the MELD and CPT scores varies in different clinical conditions. The MELD score is opined to have a better sensitivity than the CPT score in assessing the prognosis of patients with liver cirrhosis in a critical care setting. ${ }^{18}$ In our study we did not see a correlation of mortality using the CPT score in patients with liver cirrhosis.

Besides the higher mortality rate in patients with CDI and liver cirrhosis, we also noticed a difference in the mortality pattern in patients with liver cirrhosis and CDI (Figure 1). CDI tends to have an aggressive course in patients with liver cirrhosis compared to those with no liver cirrhosis, leading to early mortality in the course of disease. The higher mortality in patients with liver cirrhosis may have contributed to the shorter length of stay. This data can be utilized to follow-up on antibiotic response for patients with CDI and decide on an early escalation of therapy.

Our study has several limitations. The study is a singlecenter retrospective review. We have utilized the CDI severity criteria that were in effect in 2013, until the most recent recommendations changed the management protocol. We had a smaller group of patients with liver cirrhosis and hence had low power in concluding the impact of the MELD score on CDI-related mortality. The lower number of the liver cirrhosis patients also limited us from analyzing the impact of liver cirrhosis on mortality rates in mild CDI infections.

\section{Conclusion}

CDI does impact the mortality outcome in patients with liver cirrhosis. Patients with liver cirrhosis tend to present with a clinically severe to complicated CDI compared to those with no liver cirrhosis. However, liver cirrhosis is an independent predictor of mortality irrespective of the CDI severity. Patients with a higher MELD score in liver cirrhosis tend to have poor outcomes with CDI infection. Further studies are needed for better understanding of the clinical course of CDI in cirrhosis and to evaluate the need for early intervention in this group of patients.

\section{Disclosure}

The authors report no conflicts of interest in this work.

\section{References}

1. George RH, Symonds JM, Dimock F, et al. Identification of Clostridium difficile as a cause of pseudomembranous colitis. $B M J$. 1978;1:695. doi:10.1136/bmj.1.6114.695

2. McDonald LC, Gerding DN, Johnson S, et al. Clinical practice guidelines for Clostridium difficile infection in adults and children: 2017 update by the Infectious Diseases Society of America (IDSA) and Society for Healthcare Epidemiology of America (SHEA). Clin Infect Dis. 2018;66:e1-e48. doi:10.1093/cid/cix 1085

3. Lessa FC, Mu Y, Bamberg WM, et al. Burden of Clostridium difficile Infection in the United States. $N$ Engl J Med. 2015;372(9):825-834. doi:10.1056/NEJMoa1408913

4. Dubberke ER, Olsen MA. Burden of Clostridium difficile on the healthcare system. Clin Infect Dis. 2012;55:S88-S92. doi:10.1093/ $\mathrm{cid} / \mathrm{cis} 335$

5. Ghantoji SS, Sail K, Lairson DR, DuPont HL, Garey KW. Economic healthcare costs of Clostridium difficile infection: a systematic review. J Hosp Infect. 2010;74:309-318. doi:10.1016/j.jhin.2009.10.016

6. Gujja D, Friedenberg FK. Predictors of serious complications due to Clostridium difficile infection. Aliment Pharmacol Ther. 2009;29:635-642. doi:10.1111/j.1365-2036.2008.03914.x

7. Ofori E, Ramai D, Dhawan M, Mustafa F, Gasperino J, Reddy M. Community-acquired Clostridium difficile: epidemiology, ribotype, risk factors, hospital and intensive care unit outcomes, and current and emerging therapies. J Hosp Infect. 2018;99:436-442. doi:10.1016/ j.jhin.2018.01.015 
8. Aloreidi K, Barakat M, Ramai D, Xie C, Aamar A, Reddy M. 1063 Clostridioides difficile infection in liver cirrhosis: a multiyear nationwide analysis. ACG. 2019;114:S603.

9. Bajaj JS, Ananthakrishnan AN, Hafeezullah M, et al. Clostridium difficileIs associated with poor outcomes in patients with cirrhosis: a national and tertiary center perspective. Am J Gastroenterol. 2010;105:106-113. doi:10.1038/ajg.2009.615

10. Dotson KM, Aitken SL, Sofjan AK, Shah DN, Aparasu RR, Garey KW. Outcomes associated with Clostridium difficile infection in patients with chronic liver disease. Epidemiol Infect. 2018;146:1101-1105. doi:10.1017/s0950268818001036

11. Surawicz CM, Brandt LJ, Binion DG, et al. Guidelines for diagnosis, treatment, and prevention of Clostridium difficile infections. $\mathrm{Am}$ J Gastroenterol. 2013;108:478-498. doi:10.1038/ajg.2013.4

12. Hong SJ, Feuerstadt P, Brandt LJ. MELD is the only predictor of short-term mortality in cirrhotic patients with C. difficile infection. Dig Liver Dis. 2019;51:275-280. doi:10.1016/j.dld.2018.07.032

13. Tandon P, Garcia-Tsao G. Bacterial infections, sepsis, and multiorgan failure in cirrhosis. Semin Liver Dis. 2008;28:26-42. doi:10.1055/ s-2008-1040319
14. Llovet JM, Bartolí R, March F, et al. Translocated intestinal bacteria cause spontaneous bacterial peritonitis in cirrhotic rats: molecular epidemiologic evidence. J Hepatol. 1998;28:307-313. doi:10.1016/ 0168-8278(88)80018-7

15. Gustot T, Durand F, Lebrec D, Vincent JL, Moreau R. Severe sepsis in cirrhosis. Hepatology. 2009;50:2022-2033. doi:10.1002/hep.23264

16. Walayat S, Martin D, Patel J, et al. Role of albumin in cirrhosis: from a hospitalist's perspective. J Community Hosp Intern Med Perspect. 2017;7:8-14. doi:10.1080/20009666.2017.1302704

17. Philips CA, Ahamed R, Rajesh S, George T, Mohanan M, Augustine P. Update on diagnosis and management of sepsis in cirrhosis: current advances. World J Hepatol. 2020;12:451-474. doi:10.4254/wjh.v12.i8.451

18. Peng Y, Qi X, Guo X. Child-Pugh versus MELD score for the assessment of prognosis in liver cirrhosis: a systematic review and meta-analysis of observational studies. Medicine. 2016;95:e2877e2877. doi:10.1097/MD.0000000000002877

\section{Publish your work in this journal}

Clinical and Experimental Gastroenterology is an international, peerreviewed, open access, online journal publishing original research, reports, editorials, reviews and commentaries on all aspects of gastroenterology in the clinic and laboratory. This journal is indexed on American Chemical Society's Chemical Abstracts Service (CAS)
The manuscript management system is completely online and includes a very quick and fair peer-review system, which is all easy to use. Visit http://www.dovepress.com/testimonials.php to read real quotes from published authors. 